\title{
DRAFT: AIR-ASSISTED ATOMIZATION AT CONSTANT MASS AND MOMENTUM FLOW RATE: INVESTIGATION OF THE AMBIENT PRESSURE INFLUENCE WITH THE SPH METHOD
}

\author{
G. Chaussonnet; S. Joshi, C. Lieber, R. Koch, H.-J. Bauer \\ Institut für Thermische Strömungsmaschinen \\ Karlsruher Institut für Technologie (KIT) \\ Kaiserstr. 12 \\ 76131 Karlsruhe, Germany
}

\author{
S. Wachter, T. Jakobs, T. Kolb \\ Institut für Technische Chemie \\ Karlsruher Institut für Technologie (KIT) \\ P.O. Box 3640 \\ 76021 Karlsruhe, Germany
}

\begin{abstract}
A twin-fluid atomizer configuration is simulated by means of the 2D weakly-compressible Smooth Particle Hydrodynamics method, and compared to experiments. The Gas-to-Liquid-Ratio, the momentum flux ratio and the velocity ratio are set constant for different ambient pressures, which leads to different gaseous flow sections. The objectives of this study are to (i) investigate the effect of ambient pressure at constant global parameters, and (ii) to verify the capability of $2 \mathrm{DSPH}$ to qualitatively predict the proper disintegration mechanism and to recover the correct evolution of the spray characteristics. The setup consists of an axial liquid jet of water fragmented by a co-flowing high-speed air stream $\left(U_{g}=80 \mathrm{~m} / \mathrm{s}\right)$ in a pressurized atmosphere up to 16 bar. The results are compared to the experiment, and presented in terms of (i) mean velocity profiles, (ii) drop size distributions and (iii) Sauter Mean Diameter of the spray. It is found that there exists an optimal pressure to minimize the mean size of the spray droplets. Finally, two new quantities related to atomization are presented: (i) the breakup activity that quantifies the number of breakup events per time and volume unit and (ii) the fragmentation spectrum of the whole breakup chain, which characterizes the cascade phenomenon in terms of probability. The breakup activity confirms the presence of the optimal pressure and the fragmentation spectrum gives information on the type of breakup, depending on the ambient pressure.
\end{abstract}

*geoffroy.chaussonnet@kit.edu

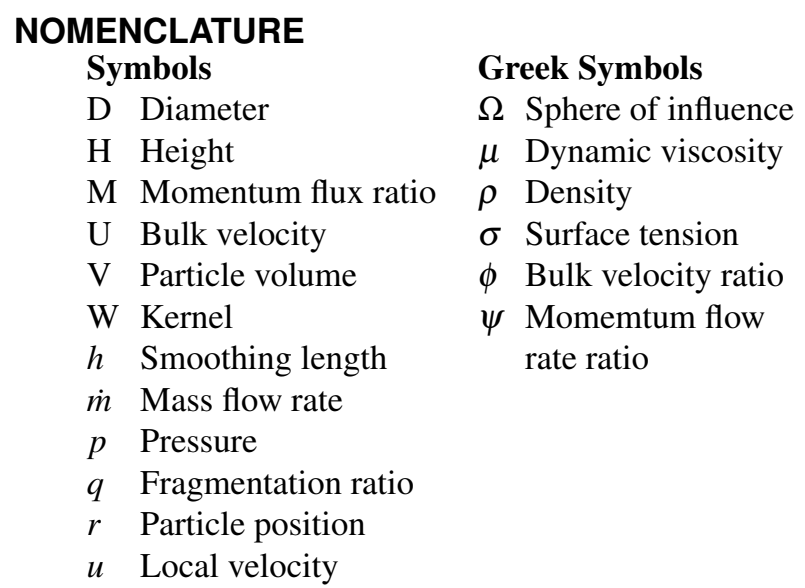

\section{INTRODUCTION}

Air-assisted atomization is investigated in the context of fuel production from biomass [1]. A key-step of this process chain is high pressure entrained flow gasification, where a high 
viscous biomass based fuel has to be atomized with a small amount of atomization agent (oxygen and steam). This biomass must be transformed into a fine spray to increase its contact surface to the gas, and in turn, to enhance the gasification reaction [2]. Due to constraints on the chemical equilibrium of the gasification reaction, typical Gas-to-Liquid-Ratio (GLR) has to be lower than one. This is challenging for atomization of high viscous liquids at system pressures up to 80 bar. Therefore, the design of the spraying nozzle is primordial to ensure a proper atomization with a constrained GLR. Some advances on entrained flow gasification were recently achieved at atmospheric pressure [3-6]. On the other hand, the influence of ambient pressure on the atomization process inside an entrained flow gasifier is a important parameter, which is still not well understood. The study of the air-assisted atomization of a highly viscous liquid at high pressure is very scarce in the literature. Sänger et al. [7] experimentally investigated the breakup process on a lab-scale geometry, while Chaussonnet et al. [8] conducted numerical simulations of the same geometry.

The objective of the present study is to numerically investigate the influence of the ambient pressure when keeping global flow parameters and velocity ratio constant. This is an original investigation, because usually the increase of pressure leads to the reduction of the gas velocity in order to conserve the global parameters. In our case, as detailed in the following, the nozzle geometry is adapted to the pressure in order to deliver the same bulk velocity. The numerical method is the Smooth Particle Hydrodynamics (SPH) method, which was originally developed for astrophysics [9] and later adapted to free surface flows [10]. It is a mesh-free Lagrangian method where the discretization elements are particles moving at the fluid velocity. The physical quantities such as pressure or density are carried and transported inside the numerical domain. Recently, this method has been successfully applied in the field of air-assisted atomization $[8,11,12]$.

The experiment is presented in a first part, followed by a description of the numerical model. The simulation results are compared to the experiment in the final part.

\section{EXPERIMENT}

The experiment is conducted at the Institute of Technical Chemistry (KIT-ITC) and consists of a twin-fluid atomizer discharging into a pressurized cavity, similar to the investigations of Sänger et al. [7]. The spray generation mechanism of this nozzle is air-assisted atomization. The liquid (blue color in Fig. 1) is injected at low velocity, and is enclosed in a coflowing highspeed air stream (green color in Fig. 1). The liquid is sharply accelerated due to the momentum diffusion from the gas, which leads to primary instabilities that further evolve to ligament and ultimately to droplets. The geometrical parameters of the nozzle exit are the diameter $D_{l}$ of the liquid duct, the height $H_{g}$ of the gas duct and the thickness $e_{s}$ of the separator. They are annotated on Fig. 1. The local operating parameters are the density $\rho$ and the bulk velocity $U$ of both phases, indexed $g$ and ${ }_{l}$ for gas and liquid, respectively. The global operating parameters are the mass and the momentum flow rate of gas and liquid injected in the cavity. They are equal to $\dot{m}=\rho U S$ and $\dot{r}=\rho U^{2} S$, respectively, where $S$ is the flowing surface.

The investigated liquid is water, with a density $\rho_{l}$, a viscosity
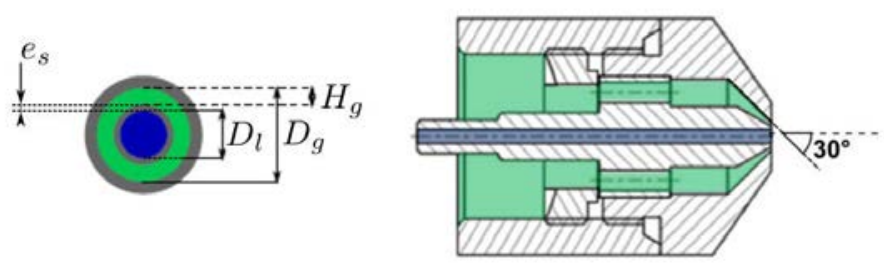

FIGURE 1: Sketch of the nozzle. Left: front view. Right: side view

$\mu_{l}$ and a surface tension $\sigma$ of $998 \mathrm{~kg} / \mathrm{m}^{3}, 1 \mathrm{mPa} \mathrm{s}$ and $72 \mathrm{~g} / \mathrm{s}^{2}$, respectively. The non-dimensional groups that characterize this setup are numerous, and the hierarchy of their influence on spray formation is still under discussion among the scientific community [13]:

$$
\mathrm{Re}=\frac{\rho D_{h} U}{\mu}, \mathrm{We}=\frac{\rho_{g} D_{l} U_{r e l}^{2}}{\sigma}, \mathrm{M}=\frac{\rho_{g} U_{g}^{2}}{\rho_{l} U_{l}^{2}}, \mathrm{GLR}=\frac{\dot{m}_{g}}{\dot{m}_{l}}
$$

In order of appearance, Eq. (1) shows (i) the Reynolds number based on the hydraulic diameter $D_{h}$ of the considered duct, (ii) the Weber number where $U_{r e l}$ is the liquid/gas relative velocity, (iii) the momentum flux ratio $\rho_{g} U_{g}^{2} / \rho_{l} U_{l}^{2}$, and (iv) the Gas-toLiquid Ratio, respectively. Note that the GLR is the only global non-dimensional group whereas $\mathrm{Re}, \mathrm{We}$, and $M$ are local quantities. This is because the GLR is a parameter for the chemical reaction of gasification. In the perspective of investigating the influence of global parameters, a new ratio is introduced, the momentum flow rate ratio $\Psi=\dot{r}_{g} / \dot{r}_{l}$ which is equal to $M \times S_{g} / S_{l}$. Finally since the atomization mechanism of this configuration relies of the momentum transfer from the gas to the liquid, the shearing of the liquid surface by the air is quantified by the bulk velocity ratio $\phi=U_{g} / U_{l}$.

The objective of this experiment is to investigate the influence of the ambient pressure when the global operating non-dimensional 
groups (GLR and $\Psi$ ) as well as the bulk velocity ratio $\phi$ are kept constant. The relationships between these parameters are:

$$
\mathrm{GLR}=\frac{\rho_{g} S_{g}}{\rho_{l} S_{l}} \phi \quad \text { and } \quad \Psi=\frac{\rho_{g} S_{g}}{\rho_{l} S_{l}} \phi^{2}
$$

In order to keep these parameters constant for different ambient pressures (i.e. different ratio $\rho_{g} / \rho_{l}$ ), it is necessary to modify the ratio $S_{g} / S_{l}$ accordingly. Therefore, the flow section of the nozzle has to be modified for each ambient pressure. The liquid duct $D_{l}$ and the separator thickness $e_{s}$ are kept constant, as well as the gas and liquid bulk velocity (Table 1 ). Only the gas height $H_{g}$ is varied according to the pressure, as summarized in Table 2. Note that the even though the global parameters are constant, the local non-dimensional parameters $\left(R e_{g}, \mathrm{We}\right.$ and $\left.\mathrm{M}\right)$ increase with the increasing pressure. The constant parameters selected for this study are:

$$
\mathrm{GLR}=0.8, \Psi=36.2, \phi=45.2
$$

TABLE 1: Constant operating parameters.

\begin{tabular}{|cccccc|}
\hline$D_{l}$ & $e_{s}$ & $U_{g}$ & $U_{l}$ & $\dot{m}_{g}$ & $\dot{m}_{l}$ \\
{$[\mathrm{~mm}]$} & {$[\mathrm{mm}]$} & {$[\mathrm{m} / \mathrm{s}]$} & {$[\mathrm{m} / \mathrm{s}]$} & {$[\mathrm{kg} / \mathrm{h}]$} & {$[\mathrm{kg} / \mathrm{h}]$} \\
2 & 0.5 & 80 & 1.77 & 16 & 20 \\
\hline
\end{tabular}

TABLE 2: Evolution of parameters with the ambient pressure

\begin{tabular}{|c|c|c|c|c|c|}
\hline Pressure & {$[\mathrm{bar}]$} & 2 & 6 & 11 & $16^{*}$ \\
Density & {$\left[\mathrm{kg} / \mathrm{m}^{3}\right]$} & 2.4 & 7.2 & 13.2 & 19.3 \\
$H_{g}$ & {$[\mathrm{~mm}]$} & 1.59 & 0.97 & 0.39 & 0.28 \\
\hline $\mathrm{Re}_{g}$ & {$[\times 1000]$} & 34.2 & 42.9 & 46.3 & 47.9 \\
$\mathrm{We}$ & {$[-]$} & 342 & 429 & 463 & 479 \\
$\mathrm{M}$ & {$[-]$} & 4.93 & 14.8 & 27.1 & 39.4 \\
\hline
\end{tabular}

* Case investigated in numerical simulations only.

The experiment was instrumented with a high-speed CCD camera and the disintegration of the liquid jet was investigated by means of backlight illumination. Laser Doppler Anemometry and Phase Doppler Anemometry (LDA/PDA) were used $40 \mathrm{~mm}$ downstream the nozzle exit to characterize the spray in terms of drop size distribution and velocity. The jet disintegration is displayed in Fig. 2. All the four cases correspond to a fiber-type breakup regime. It is observed that the liquid fibers decrease with an increasing ambient pressure, up to $p=6$ bar. Interestingly, the size of the fibers at $p=11$ bar are larger than at 6 bar.
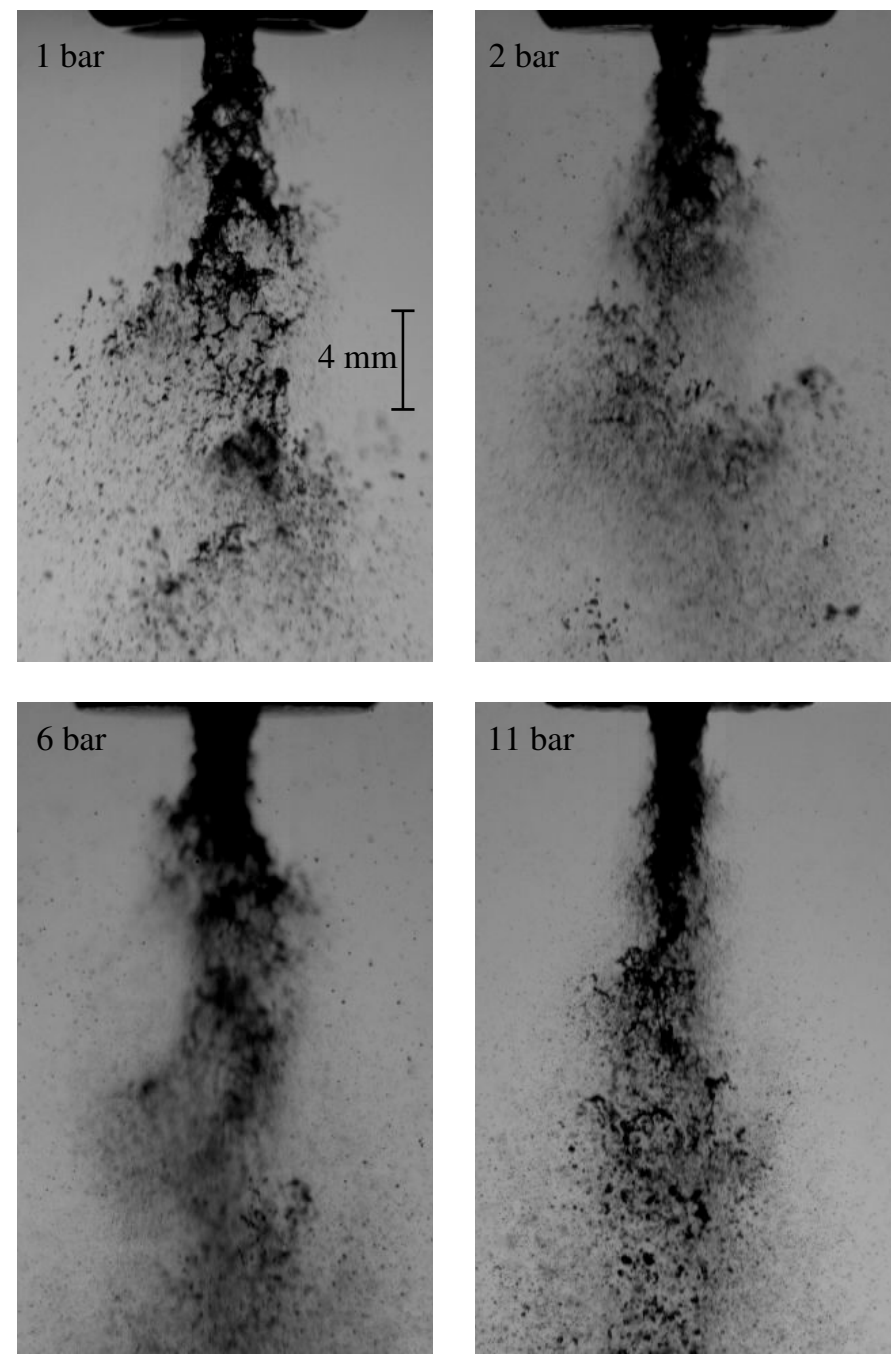

FIGURE 2: Experimental evidences of the liquid jet disintegration.

\section{NUMERICAL MODEL}

In the SPH mehod, the physical quantities and their gradients at a particle location $(a)$ are expressed by interpolating over the neighbor particles $(b)$ [14]:

$$
\begin{aligned}
f\left(\boldsymbol{r}_{a}\right) & =\sum_{b \in \Omega_{a}} V_{b} f\left(\boldsymbol{r}_{b}\right) W\left(r_{b}-r_{a}, h\right) \\
\nabla f\left(\boldsymbol{r}_{a}\right) & =\sum_{b \in \Omega_{a}} V_{b}\left[f\left(r_{b}\right)+f\left(r_{a}\right)\right] \nabla W\left(\boldsymbol{r}_{b}-r_{a}, h\right)
\end{aligned}
$$

where $V_{b}$ is the volume of the adjacent particles. The term $W$ is referred to as the kernel and promotes the influence of closer neighbors as illustrated in 2D in Fig. 3 (top). It depends on the inter particle distance $r_{b}-r_{a}$ and a characteristic length scale $h$ 
called the smoothing length. The kernel has a compact support, so that the neighbors located outside the sphere of influence $\Omega_{a}$ (Fig. 3 bottom) are not taken into account.

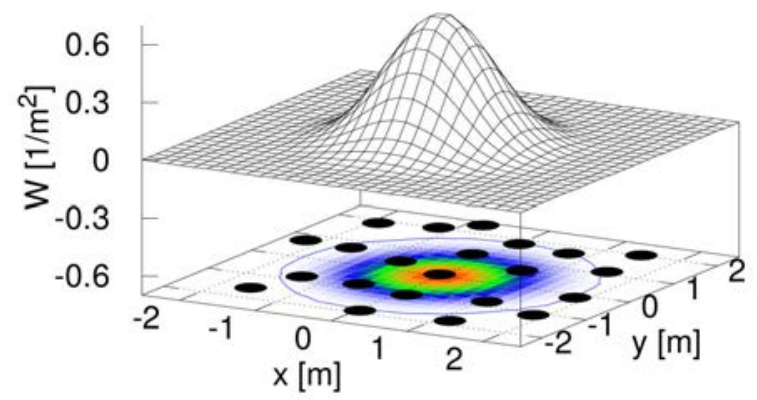

FIGURE 3: Top part: Surface of a 2-D kernel. Bottom part: Particle distribution superimposed with the kernel color map and illustration of the sphere of influence.

In the present study, the evolution of the gas and the liquid phases are described by the isothermal weakly-compressible NavierStokes equations. The source terms in the momentum conservation are the pressure gradient, the viscous term proportional to the Laplacian of the velocity, the surface tension force and the gravity. The flow is supposed weakly compressible, so that a fictive sound speed is set, lower than the real one. As the speed of sound limit the time step due to the CFL condition, the weaklycompressible approach allows to increase the time step without changing the main features of the flow. The governing equations are formulated in a Lagrangian frame of reference and solved using the SPH method. Because the simulations presented in this study are 2D, the equations are modified to take into account some 3D aspects. First, an artificial surface tension force is added to take into account the curvature of the jet when it exits the nozzle. Second, the Laplacian operator is modified to account the difference of shearing between the inner and outer surface of an infinitesimal elements. Third, the radial velocity (i.e. the vertical) is decreased to mimic the mass conservation of a fluid element moving radially. The complete numerical model cannot be presented here due to space limitation. The interested reader is referred to [8] for a complete description.

\section{NUMERICAL SETUP}

The numerical domain is a $2 \mathrm{D}$ abstraction of the real experiment. It consists of the 2D-plane crossing the center plane of the nozzle (Fig. 4). The advantage of this domain compared to a pure axisymmetric one is that the flapping of the jet can be taken into account. The $3 \mathrm{D}$ corrections presented in the previous section are introduced to model the different effects of the real geometry. In [8], it was shown that even though this numerical setup can lead to deviations in terms of dynamics of the liquid jet, the influence of the ambient pressure on the spray characteristics are well recovered.

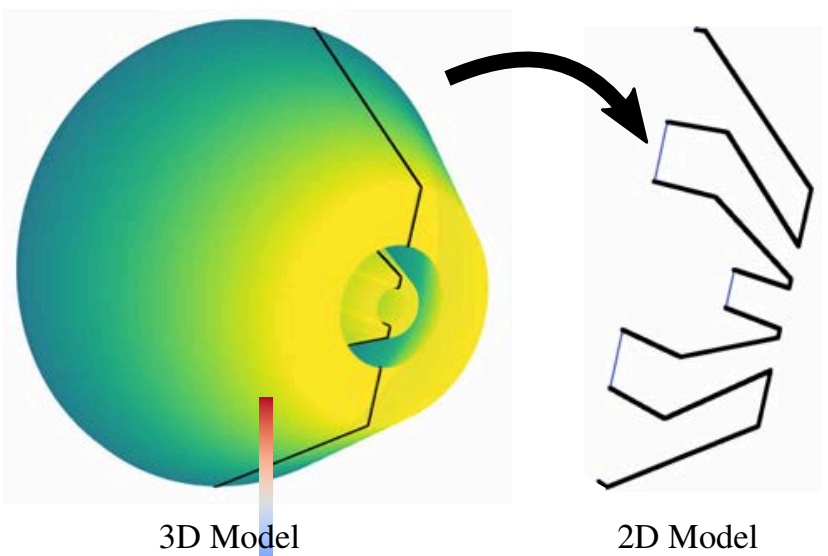

FIGURE 4: Illustration of the 2D abstraction.

The numerical domain is shown in Fig. 5. It consists of the injector and the quiescent cavity, long by $50 \mathrm{~mm}$ downstream the nozzle orifice and wide by $60 \mathrm{~mm}$. At the gas and liquid inlets a turbulent profile is imposed. The bulk velocity is imposed so that $U_{g}=80 \mathrm{~m} / \mathrm{s}$ and $U_{l}=1.77 \mathrm{~m} / \mathrm{s}$ at the nozzle exit. The lengths of the inlet ducts are at least five time the ducts height. A no-slip boundary condition [15] is imposed on the nozzle walls. The pressurized cavity in the experiment is larger than the numerical domain. Hence, a slip condition is imposed on the walls of the cavity to cancel any boundary layer effects far away from the nozzle. A constant pressure condition is imposed at the outlet. An entrainment stream of $7.5 \mathrm{~m} / \mathrm{s}$ is imposed on the sides of the nozzle (i) to mimic the entrainment of the jet, (ii) to reduce the influence of the artificial recirculation zones in the cavity and (iii) to ensure a steady outflow velocity at the outlet. Both inlet and outlet conditions are implemented to damp reflected pressure waves, as detailed in [16]. The fictive sound speed is set to $342 \mathrm{~m} / \mathrm{s}$ for the gas and $45 \mathrm{~m} / \mathrm{s}$ for the liquid. Note that in this case the gas sound speed corresponds to the real value, thus accounting for compressible effects.

The same nozzle was simulated with different gas flow sections depending on the ambient pressure. The modification of $H_{g}$ is illustrated in Fig. 6.

The space discretization is given by the mean inter particle distance of $12.5 \mu \mathrm{m}$, which leads to 23.5 million particles. According to a previous study [8], this discretization is enough resolve the volume Probability Density Function (PDF) of the spray. At the beginning of the simulation, the cavity is filled with gas particles. The simulations are run for a physical time of 


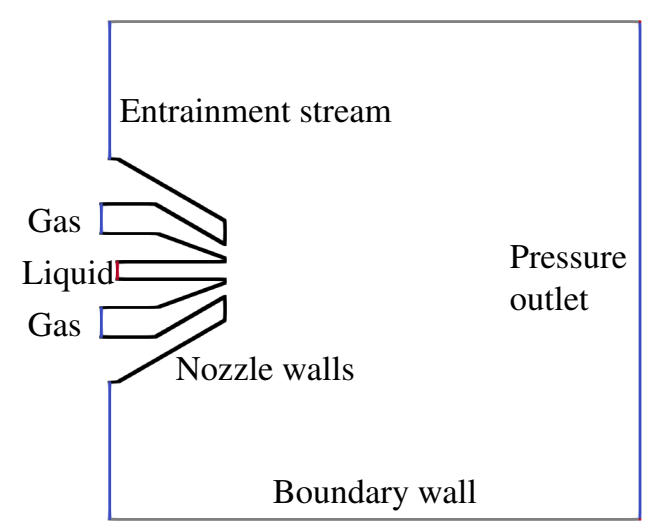

FIGURE 5: Sketch of the numerical domain.
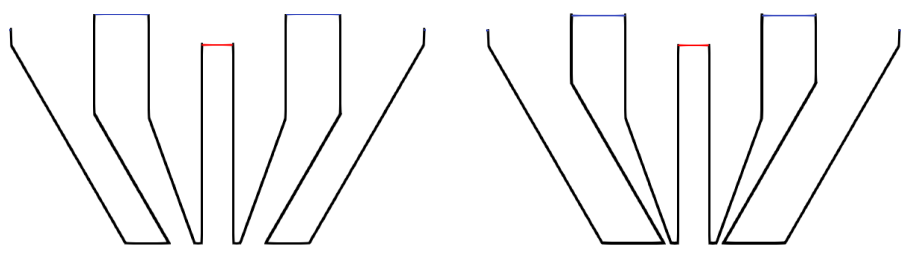

FIGURE 6: Closeup view of the nozzle for 2 (left) and 11 (right) bar.

$50 \mathrm{~ms}$ on 1920 processors. This leads to 18 convective times, ensuring converged statistics for the gas flow. The mean time step $\Delta t$ is $20 \mathrm{~ns}$, and the instantaneous solutions are exported at a rate of $21.5 \mathrm{kHz}$.

\section{RESULTS AND DISCUSSION Instantaneous solutions}

Figure 7 represents a superposition of three instantaneous snapshots for an ambient pressure of 11 bar, colored by the mean liquid volume fraction $\alpha_{l}$. It was chosen to superimpose several snapshots to qualitatively compare with the experiment. Indeed, the experimental image represent a full 3D configuration whereas one snapshot from the 2D simulation consists of a plane only. Therefore, to qualitatively represent as much liquid in the simulation as in the experiment, several snapshots were superimposed. Comparing Figs. 2 and 7 show that the spray structure is well recovered by the numerical simulation. However, the intact length is strongly underestimated in the simulation. The spray angle is also overestimated, due to a too large flapping phenomenon compared to the experiment.

\section{Time-averaged quantities}

The global features of the mean flow are depicted in Fig. 8, where the mean velocity is superimposed with the streamlines issued from the inlets. Both gas and liquid particles were used to

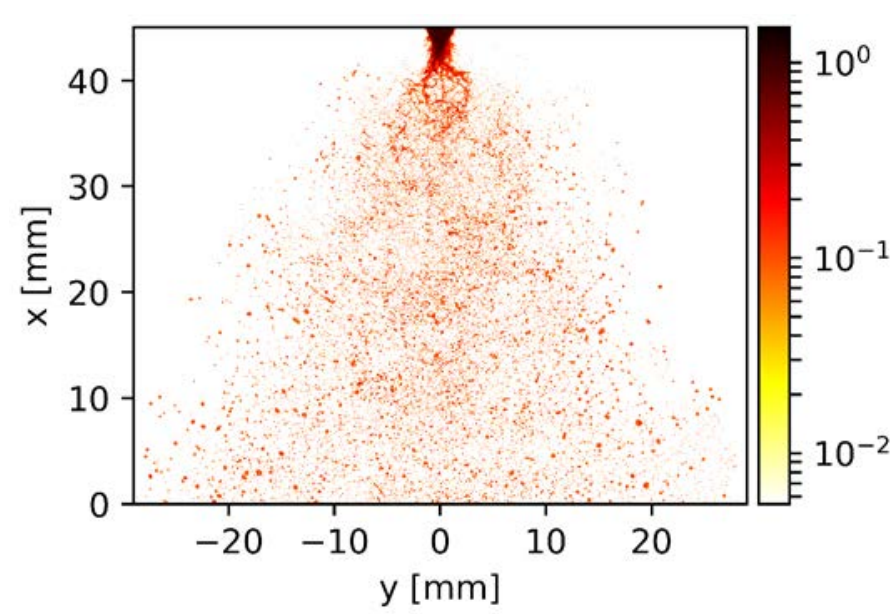

FIGURE 7: Superposition of instantaneous snapshots.

compute the axial velocity. The acceleration of the gas in the duct upstream of the nozzle exit as well as the opening angle of the turbulent gaseous jet are visible. The streamlines illustrate very well the entrainment flux due to the high speed air stream. Due to the entrainment inlets, no strong recirculation zone is observed. It is to be noted that the high velocity zones induced by the two air streams join at the centerline, at $x \approx 4 \mathrm{~mm}$. This means that the liquid is strongly accelerated in this region

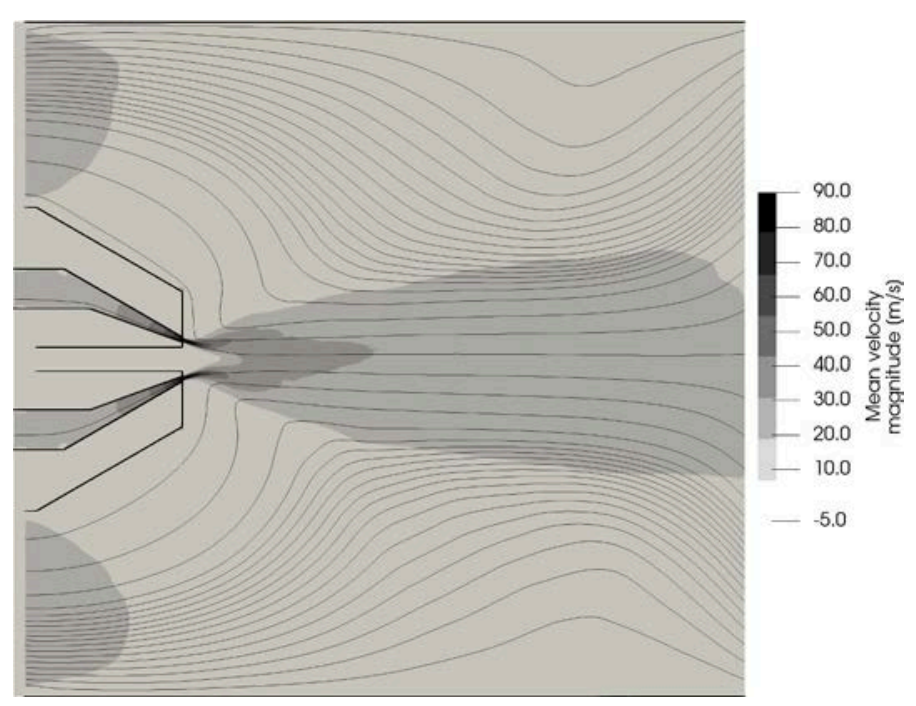

FIGURE 8: Mean axial velocity superimposed with the streamlines.

To monitor the time-average of the spray quantities (volume fraction $\alpha_{l}$, droplet concentration $N_{d}$, Sauter Mean Diameter), the liquid particles are projected onto a Cartesian background grid and averaged over the time. The result is depicted in Fig. 9 
for 11 bar. First of all, it is visible in the outer part of the spray that the statistical convergence is not reached. Indeed, some large liquid blobs are accounted several time along their motion towards the exit, which leads to depict the trajectory of such structures. Nevertheless, the inner part of the spray is regular and well converged. At the nozzle exit, the intact liquid jet is visible with a mean value equal to one. The early detachment of small droplets is visible with a very low $\alpha_{l}$ around the liquid jet at $0<\mathrm{x}<5 \mathrm{~mm}$. At $x \approx 5 \mathrm{~mm}$, the flapping of the jet is visible with a distribution of $\alpha_{l}$ in the shape of a trumpet opening.

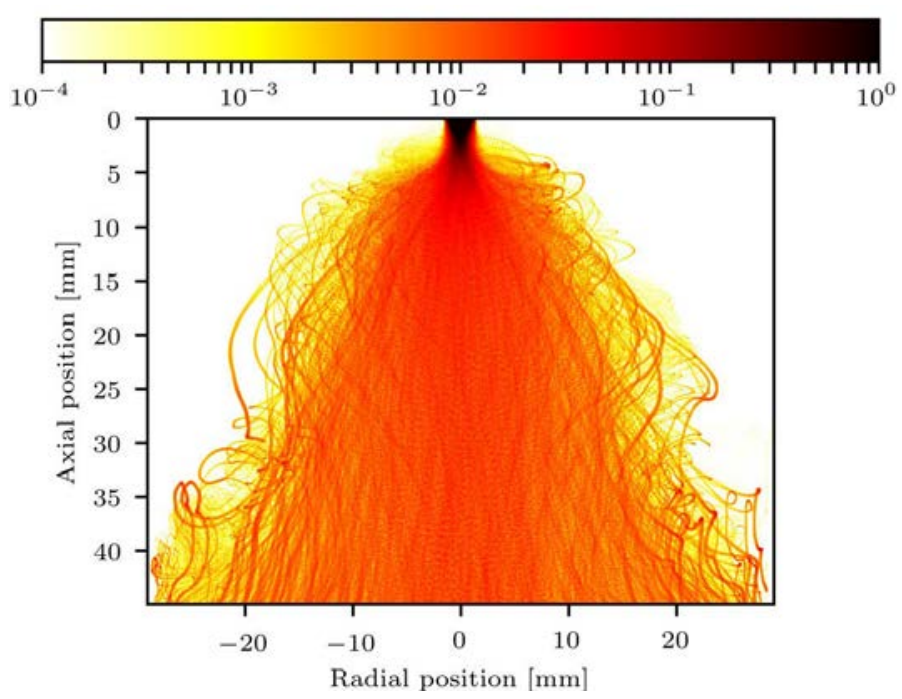

FIGURE 9: Mean liquid volume fraction $\alpha_{l}$ for $\mathrm{p}=11 \mathrm{bar}$.

\section{Spray characteristics}

In the experiment, the spray characteristics are measured with LDA/PDA on a plane located at $x=40 \mathrm{~mm}$. In the numerical simulation, the spray characteristics are collected on a slice located at $x$ between 35 and $45 \mathrm{~mm}$. It is to be noted that due to the proximity of the measuring device to the nozzle exit $(40 \mathrm{~mm})$, the spray droplets were not all spherical and the rejection rate due to unvalid sphericity was significant. Therefore, in order to ensure a fair comparison between the experiment and the simulation, simulated droplets with a sphericity lower than 95\% were dismissed. This lead to dismiss between 5 and $10 \%$ of the total droplets in the collecting region. This data set is referred to as filtered droplets in the following. On the other hand, the whole data set corresponds to a more realistic spray, and it is labeled unfiltered droplets. The local Sauter Mean Diameter (SMD) is plotted versus the radial coordinate in Fig. 10 (top). For a pressure of 11 bar, the agreement is very good in terms of trends and absolute value. For $p=2$ and 6 bar, the agreement is acceptable for $y<10 \mathrm{~mm}$. Larger $y$ coordinate lead to an strong increase of the SMD in the numerical simulations whereas it is rather constant in the experiment. This can be explained by the flapping of the jet in the simulation, which eject larger liquid lumps, whereas the experiment does not exhibit a strong flapping. The axial velocity is plotted versus $y$ in Fig. 10 (bottom). As in the experiment, the velocity increases in the center of the spray, and the effect is stronger with a lower ambient pressure. However, the absolute value of the maximum velocity is not well predicted, most presumably due to the $2 \mathrm{D}$ geometry.
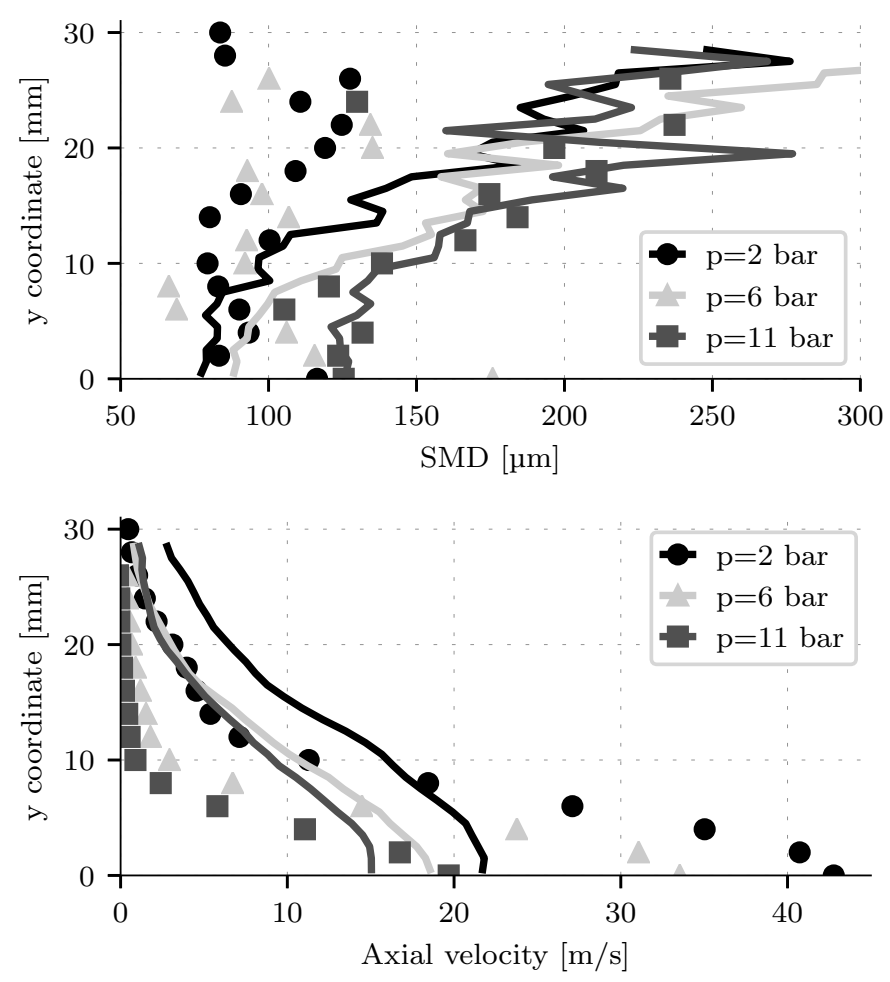

FIGURE 10: Evolution of the SMD (top) and the axial velocity (bottom) in the radial direction at the probing slice. Experiment and simulation are represented by symbols and lines, respectively.

The global SMD is plotted versus the pressure for the filtered and unfiltered droplets in Fig. 11. Concerning filtered droplets, the SMD follows the same trend as with the experiment, i.e. a rather constant SMD between 2 and 6 bar, and a larger SMD for 11 bar. Concerning the non-filtered droplets, the SMD at 2 bar is larger than at 11 and 16 bar, and it is minimal at 6 bar. This suggests that the rejection of the non-spherical droplets by the LDA/PDA technique leads to a larger bias at $p=2$ bar compared to 11 bar.

The striking results, highlighted by experiment and numerical simulation, is that there exists an optimal pressure in order to ob- 
tain the smallest SMD, at constant GLR, $\psi$ and $\phi$. This means that for a given injected mass and momentum of gas, at the same bulk velocity, the quality of the atomization depends on the ambient pressure and the gas flow section of the nozzle. Therefore, the global parameters (GLR, $\psi$ and $\phi$ ) are not sufficient to determine the spray quality. The same comments applied for the local non-dimensional numbers ( $\mathrm{Re}, \mathrm{We}$ and $\mathrm{M}$ in Eq.1). Table 2 recalls that in this configuration, $\mathrm{Re}$, We and $\mathrm{M}$ increase with the pressure. This implies that there is also an optimal value for the non-dimensional numbers to obtain the finest spray. This observation could be explained as follow. At high pressure, the air duct is so thin, that most of the air momentum is transferred to the liquid at the first impact of the gas stream on the jet surface. Since the momentum flux is large, this generates a local cloud of fine droplets from the outer part of the jet, leaving the inner jet core intact and dissipating most of the gas kinetic energy. Downstream, the momentum of the air stream is too weak to trigger the primary instabilities on the remaining liquid jet. At lower pressure, the air stream is wider, which ensure a more homogeneous momentum transfer to the liquid and leads to larger primary instabilities.

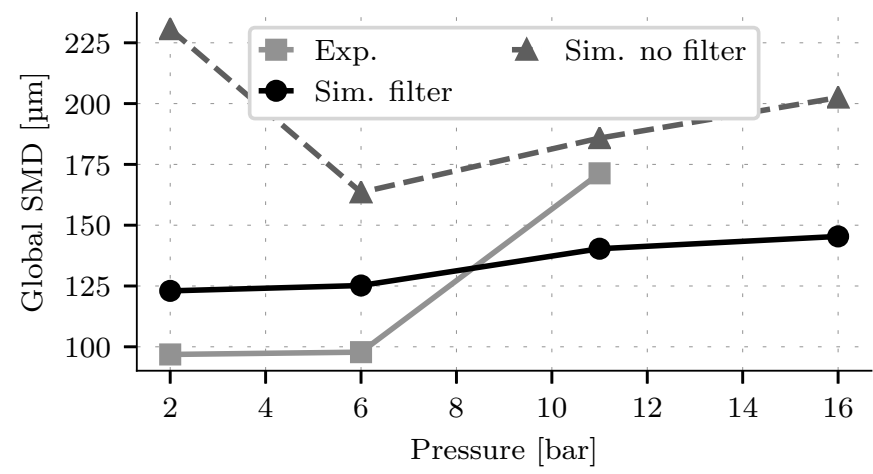

FIGURE 11: Global SMD versus ambient pressure

Figure 12 shows the volume Probability Density Function (PDF) of the spray inside the collecting region, for unfiltered droplets. The general shape of the PDF is similar to the distributions usually encountered in this type of application. For $p=6$ bar, the peak is slightly shifted towards low diameters and the distribution is significantly wider at 2 bar.

\section{Tree of fragmentation}

Due to the Lagrangian aspect of the SPH method, all numerical particles are tracked during the simulation. For liquid particles, the cluster (i.e. the droplet) they belong to can be monitored at each time step. Therefore, it is possible to record the whole genealogy of droplets that contained a given particle dur-

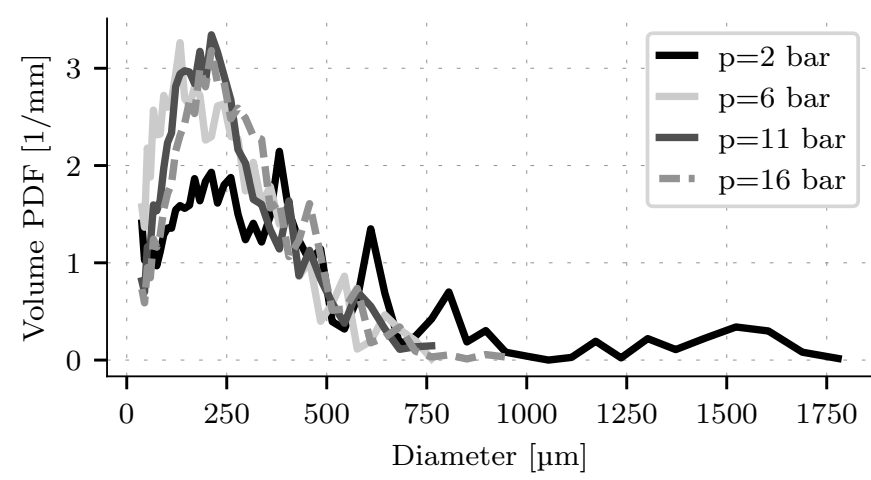

FIGURE 12: Volume PDF of the spray at the probing slice without filter on sphericity.

ing the simulation. This can be regarded as a causal tree (Fig. 13) and it is referred to as the tree of fragmentation in the following.
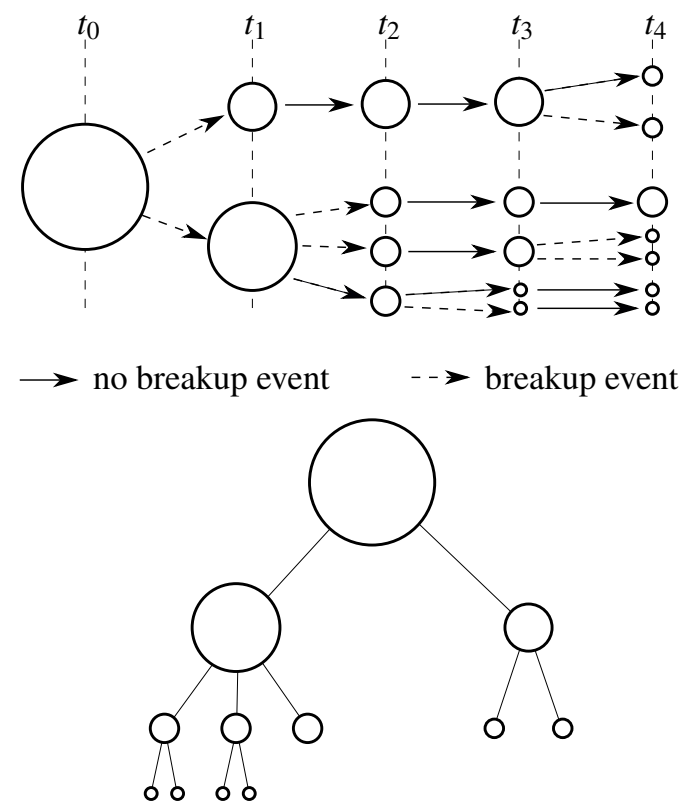

FIGURE 13: Sketch of the tree of fragmentation, from [17].

From this tree structure, the breakup activity $N_{\phi}$ can be determined. It is defined as the number of breakup events per time and volume unit. Its location is defined as the average coordinates between the mother drop and the fragmented droplets. When $N_{\phi}$ is zero, it means that no breakup occurs, and therefore that the spray is in a stable form. With respect to industrial applications such as combustion, this quantity is of interest for preliminary study because it could determines the location where to ignite the spray. 
Figure 14 shows the profile of $N_{\phi}$ versus the axial position. The region with the largest breakup activity is at $x=10 \mathrm{~mm}$, directly downstream the intact jet. The ambient pressure with largest breakup activity is 6 bar, which corroborates the experimental and numerical data on the spray characteristics. At the outlet of the domain $(x=50 \mathrm{~mm})$, the breakup phenomenon is still active $\left(N_{\phi}>0\right)$, suggesting that the sprays is not in a stable state at the outlet.

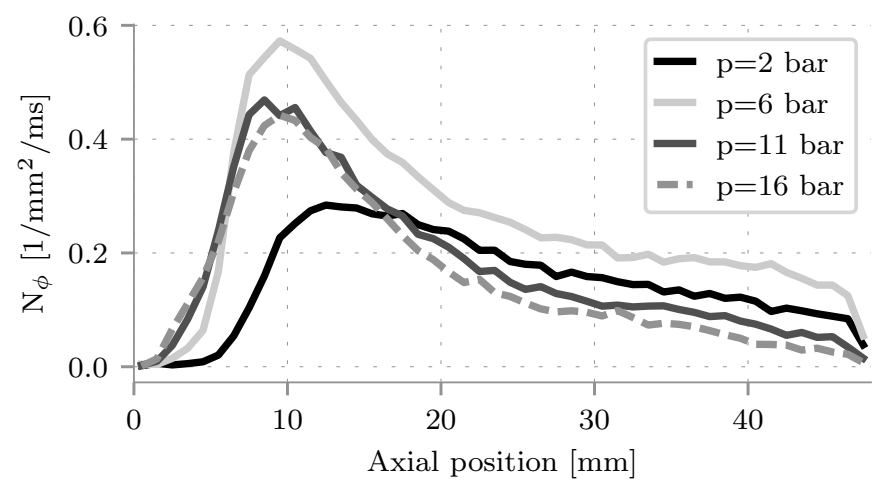

FIGURE 14: Axial profile of the breakup activity $N_{\phi}$.

One more interesting quantity can be extracted from the tree of fragmentation, the fragmentation spectrum. It is expressed as follow. The ratio of child-to-mother droplet diameter $q=D_{\text {child }} / D_{\text {mother }}$ is computed for every breakup event and collected in a statistical set. The PDF of this set determines the probability to obtain a breakup event of a given ratio. Figure 15 (top) displays the whole PDF of $q$. The zone with $q<1$ corresponds to breakup regimes while $q>1$ corresponds to coalescence. Most of the events occur in the breakup regime. With two distinct slopes, the two regimes show distinct behaviors. As their trend line is linear in a log-log plot, their expression is of the form $y=A x^{B}$. Note that this form of fragmentation spectrum was also suggested by Brown [18]. Finally, it is observed that the pressure has no influence on the fragmentation spectrum. Gorokhovski \& Saveliev [19] studied the kinetic evolution of the fragmentation spectrum in the long time limit, i.e. for a large Weber number. They showed in [20] that the ratio of the two first logarithmic moments of $q$ depends on the wavelength of the primary instabilities, which in turn, depend on the Weber number. According to Table 2, for a pressure between 6 and 16 bar, the Weber number lies between 429 and 479. This means that a weak variation of the Weber number shows almost no influence on the fragmentation spectrum. This conclusion is consistent with the theory of Gorokhovski and coworkers.

Some authors [19] restrain the PDF to the domain $q<1$, which corresponds to the breakup regime only, as illustrated in Fig. 15 (bottom). At 2 bar, the spectrum is slightly stepper than for the others, suggesting breakup events with smaller $q$ ratio. This corresponds to a breakup regime closer to shearing breakup or peeling-off breakup, where very small lumps are torn apart from a much larger liquid structure.
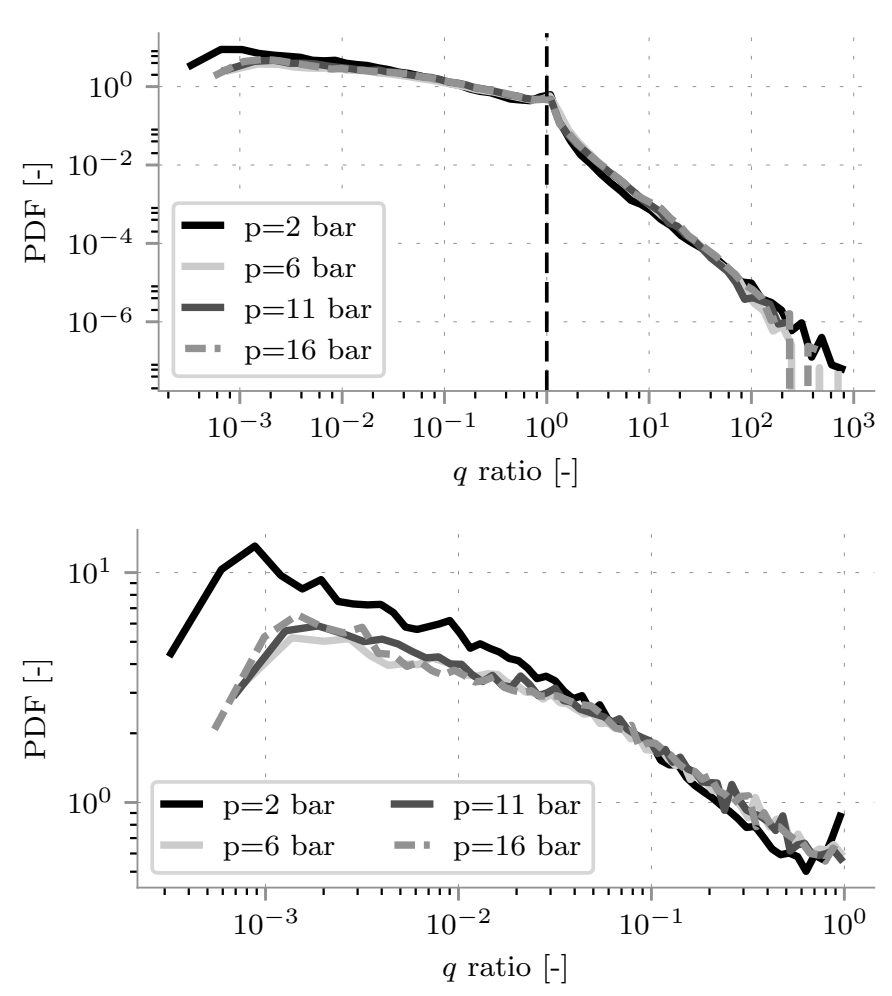

FIGURE 15: Whole fragmentation spectrum (top) and restricted to $q<1$ (bottom).

\section{CONCLUSION}

In this study, the air-assisted atomization of a water jet was simulated in a 2D geometry by means of the SPH method and compared to experiment. The effect of the ambient pressure was investigated while keeping constant the GLR, the momentum flow rate ratio and the velocity ratio. This led to use a different gas flow section for different pressures, with the same nozzle design. The numerical simulations showed that there exists an optimal ambient pressure (6 bar in this case) to obtain the smallest SMD. These results were confirmed by the experiment, even though the predicted SMD deviates from the experiment. The existence of the optimal pressure is explained by the concentration of momentum of the gas impacting the liquid jet, turning a small amount of liquid into fine droplets, but leaving intact the central core of the jet. It was shown that the use of the SPH method to investigate spray generation enables to extract 
advanced spray quantities such as the breakup activity and the fragmentation spectrum. The former is of interest for predesign studies of new nozzles, while the latter could bring some missing knowledge in the fundamental understanding of liquid atomization.

\section{ACKNOWLEDGMENT}

This work was performed on the computational resource ForHLR Phase II funded by the Ministry of Science, Research and the Arts Baden-Württemberg and DFG ("Deutsche Forschungsgemeinschaft"). The Authors like to thank the Helmholtz Association of German Research Centres (HGF) for funding (Grant: 34.14.02).

\section{References}

[1] Dahmen, N., Dinjus, E., Kolb, T., Arnold, U., Leibold, H., and Stahl, R., 2012. "State of the art of the bioliq process for synthetic biofuels production". Environmental Progress \& Sustainable Energy, 31(2), pp. 176-181.

[2] Jakobs, T., Djordjevic, N., Fleck, S., Mancini, M., Weber, R., and Kolb, T., 2012. "Gasification of high viscous slurry $\mathrm{R} \& \mathrm{D}$ on atomization and numerical simulation". Applied energy, 93, pp. 449-456.

[3] Zheng, J.-L., Zhu, Y.-H., Zhu, M.-Q., Wu, H.-T., and Sun, R.-C., 2018. "Bio-oil gasification using air - steam as gasifying agents in an entrained flow gasifier". Energy, 142, pp. $426-435$.

[4] Fleck, S., Santo, U., Hotz, C., Jakobs, T., Eckel, G., Mancini, M., Weber, R., and Kolb, T., 2018. "Entrained flow gasification part 1: Gasification of glycol in an atmospheric-pressure experimental rig". Fuel, 217, pp. 306 -319 .

[5] Mancini, M., Alberti, M., Dammann, M., Santo, U., Eckel, G., Kolb, T., and Weber, R., 2018. "Entrained flow gasification. part 2: Mathematical modeling of the gasifier using rans method". Fuel, 225, pp. 596-611.

[6] Eckel, G., Clercq, P. L., Kathrotia, T., Saenger, A., Fleck, S., Mancini, M., Kolb, T., and Aigner, M., 2018. "Entrained flow gasification. part 3: Insight into the injector near-field by large eddy simulation with detailed chemistry". Fuel, 223, pp. $164-178$.

[7] Sänger, A., Jakobs, T., Djordjevic, N., and Kolb, T., 2015. "Experimental investigation on the influence of ambient pressure on twin-fluid atomization ofliquids with various viscosities". In Proceedings of the Triennal International Conference on Liquid Atomization and Spray System (ILASS).

[8] Chaussonnet, G., Koch, R., Bauer, H.-J., Sänger, A., Jakobs, T., and Kolb, T., 2018. "Smoothed particle hydrodynamics simulation of an air-assisted atomizer operat- ing at high pressure: Influence of non-newtonian effects". Journal of Fluids Engineering, 140(6), p. 061301.

[9] Gingold, R., and Monaghan, J. J., 1977. "Smoothed particle hydrodynamics-theory and application to non-spherical stars". Mon. Not. R. Astron. Soc., 181, pp. 375-389.

[10] Monaghan, J. J., 1994. "Simulating free surface flows with SPH”. J. Comput. Phys., 110(2), pp. 399-406.

[11] Koch, R., Braun, S., Wieth, L., Chaussonnet, G., Dauch, T., and Bauer, H.-J., 2017. "Prediction of primary atomization using smoothed particle hydrodynamics". European Journal of Mechanics - B/Fluids, 61(Part 2), pp. 271 - 278. Rotating Flows.

[12] Pereira, G. G., Cleary, P. W., and Serizawa, Y., 2018. "Prediction of fluid flow through and jet formation from a high pressure nozzle using smoothed particle hydrodynamics". Chemical Engineering Science, 178, pp. 12-26.

[13] Dumouchel, C., 2008. "On the experimental investigation on primary atomization of liquid streams". Experiments in Fluids, 45, pp. 371-422.

[14] Monaghan, J. J., 2005. "Smoothed particle hydrodynamics". Reports on Progress in Physics, 68, pp. 1703 - 1759.

[15] Takeda, H., Miyama, S., and Sekiya, M., 1994. "Numerical simulation of viscous flow by smoothed particle hydrodynamics". Progress of Theoretical Physics, 92(5), pp. $939-$ 960.

[16] Braun, S., Wieth, L., Koch, R., and Bauer, H.-J., 2015. "A framework for permeable boundary conditions in SPH: Inlet, outlet, periodicity". In Proc. of the 10th Internat. SPHERIC Workshop.

[17] Chaussonnet, G., Braun, S., Dauch, T., Keller, M., Sänger, A., Jakobs, T., Koch, R., Kolb, T., and Bauer, H.-J. "Toward the development of a virtual spray test-rig using the smoothed particle hydrodynamics method". Under review at Computers \& Fluids, submitted in March 2018.

[18] Brown, W. K., 1989. "A theory of sequential fragmentation and its astronomical applications". Journal of Astrophysics and Astronomy, 10(1), pp. 89-112.

[19] Gorokhovski, M., and Saveliev, V., 2008. "Statistical universalities in fragmentation under scaling symmetry with a constant frequency of fragmentation". Journal of Physics D: Applied Physics, 41(8), p. 085405.

[20] Gorokhovski, M., Jouanguy, J., and Chtab, A., 2006. "Simulation of air-blast atomization: 'floating guard'statistic particle method for conditioning of les computation; stochastic models of break-up and coalescence". In Proc. Int. Conf. Liq. Atom. Spray Syst., 10th (ICLASS-2006). 
Karlsruher Institut für Technologie

\section{Repository KITopen}

Dies ist ein Postprint/begutachtetes Manuskript.

\section{Empfohlene Zitierung:}

Chaussonnet, G.; Joshi, S.; Wachter, S.; Koch, R.; Jakobs, T.; Kolb, T.; Bauer, H.-J. Air-Assisted Atomization at Constant Mass and Momentum Flow Rate: Investigation of the Ambient Pressure Influence with the SPH Method.

2019. Proceedings of the ASME Turbo Expo 2019: Turbomachinery Technical Conference and Exposition (GT2019)

doi:10.5445/IR/1000097128

Zitierung der Originalveröffentlichung:

Chaussonnet, G.; Joshi, S.; Wachter, S.; Koch, R.; Jakobs, T.; Kolb, T.; Bauer, H.-J. Air-Assisted Atomization at Constant Mass and Momentum Flow Rate: Investigation of the Ambient Pressure Influence with the SPH Method.

2019. Proceedings of the ASME Turbo Expo 2019: Turbomachinery Technical Conference and Exposition (GT2019), The American Society of Mechanical Engineers (ASME).

doi:10.1115/GT2019-90372 